\title{
Do telemedical interventions improve quality of life in patients with COPD? A systematic review
}

\author{
This article was published in the following Dove Press journal: \\ International Journal of COPD \\ 21 April 2016 \\ Number of times this article has been viewed
}

\author{
Thorbjørn L Gregersen' \\ Allan Green' \\ Ejvind Frausing' \\ Thomas Ringbæk ${ }^{1,2}$ \\ Eva Brøndum' \\ Charlotte Suppli Ulrik ${ }^{1,2}$ \\ 'Department of Pulmonary \\ Medicine, Hvidovre Hospital, \\ Hvidovre, ${ }^{2}$ Institute of Clinical \\ Medicine, University of Copenhagen, \\ Copenhagen, Denmark
}

\begin{abstract}
Objective: Telehealth is an approach to disease management, which may hold the potential of improving some of the features associated with COPD, including positive impact on disease progression, and thus possibly limiting further reduction in quality of life (QoL). Our objective was, therefore, to summarize studies addressing the impact of telehealth on QoL in patients with COPD.

Design: Systematic review.

Methods: A series of systematic searches were carried out using the following databases: PubMed, EMBASE, Cochrane Controlled Trials Register, and ClinicalTrials.gov (last updated November 2015). A predefined search algorithm was utilized with the intention to capture all results related to COPD, QoL, and telehealth published since year 2000.

Outcome measures: Primary outcome was QoL, assessed by validated measures.

Results: Out of the 18 studies fulfilling the criteria for inclusion in this review, three studies found statistically significant improvements in QoL for patients allocated to telemedical interventions. However, all of the other included studies found no statistically significant differences between control and telemedical intervention groups in terms of QoL.

Conclusion: Telehealth does not make a strong case for itself when exclusively looking at QoL as an outcome, since statistically significant improvements relative to control groups have been observed only in few of the available studies. Nonetheless, this does not only rule out the possibility that telehealth is superior to standard care with regard to other outcomes but also seems to call for more research, not least in large-scale controlled trials.
\end{abstract}

Keywords: COPD, telehealth care, quality of life, review, telemedicine

\section{Introduction}

Patients suffering from COPD normally follow a disease trajectory that has a progressive worsening of their lung capacity as an essential characteristic. ${ }^{1}$ In the wake of disease progression, gradually impaired physical functioning, emotional functioning, and social functioning are typically seen, resulting in a concomitant reduction in quality of life (QoL). ${ }^{2}$ In addition, owing to the nature of the disease, a well-documented high prevalence of psychological distress is found among patients with COPD, further adding to the severe adverse effects on their QoL. ${ }^{3,4}$ Measures reinforcing disease control and slowing disease progression are therefore persistently sought. Another motivation for seeking out new approaches to disease management is the fact that COPD is one of the main causes of morbidity and mortality worldwide, significantly increasing global health expenditures. ${ }^{5,6}$ Especially, exacerbations of the disease have been shown to both negatively impact patient prognosis and add to the direct costs of COPD.

One new approach to disease management is telehealth interventions in different forms and modes of application. Applying these technologies to patients with COPD has
Correspondence: Charlotte Suppli Ulrik Department of Pulmonary Medicine, Hvidovre Hospital, Kettegård Alle 30, DK-2650 Hvidovre, Denmark Email csulrik@dadlnet.dk (c) (7) (2) 2016 Gregersen et al. This work is published and licensed by Dove Medical Press Limited. The full terms of this license are available at https://www.dovepress. com/terms.php
and incorporate the Creative Commons Attribution - Non Commercial (unported, v3.0) License (http://creativecommons.org /licenses/by-nc/3.0/). By accessing the work you hereby accept the Terms. Non-commercial uses of the work are permitted without any further (ermission from Dove Medical Press Limited, provided the work is properly attributed. For permission for commercial use of this work, please see paragraphs 4.2 and 5 of our Terms (https://www.dovepress.com/terms.php). 
included 1) monitoring of vital signs, potentially foreseeing and avoiding exacerbations, 2) patient education, potentially improving patient self-management, and 3) pulmonary rehabilitation, especially in sparsely populated areas. Additionally, every new application in itself holds the promise of reduced health care costs. It is important to note, however, that the political popularity of telehealth care rests on the hope invested in the potentials rather than the proven merits of the new technologies. ${ }^{5,7}$

This review seeks to cast some more light on a corner of this emerging health industry by examining the scientific literature concerning the effects of telemedical interventions on QoL in patients diagnosed with COPD. Thus, the task here has not been to evaluate the viability of telemedicine in terms of cost-effectiveness but instead to explore whether these technologies carry any benefits in a humanistic perspective, in terms of QoL, when compared to current standard care.

\section{Methods}

\section{Search strategy}

The general principles of the Preferred Reporting Items for Systematic reviews and Meta-Analyses (PRISMA) guidelines ${ }^{8}$ were adopted to perform this review. A series of systematic searches was carried out, last updated November 2015, using the databases PubMed, EMBASE, Cochrane Controlled Trials Register, and ClinicalTrials.gov. The strategy was to assemble as much literature about telehealth care interventions applied to patients with COPD as possible. In order to do that, the search algorithm consisted of whole words, short terms, and exact chosen order of words (with the use of " " symbols) combined with MeSH terms, and the searches were therefore carried out using the following algorithm: ("copd" OR "cold" OR "coad" OR “chronic obstructive pulmonary disease" OR "chronic obstructive lung disease" OR "chronic obstructive airway disease" OR “chronic airflow obstruction" OR "chronic bronchitis" OR "pulmonary emphysema") AND ("telehealth" OR "telemedicine" OR "eHealth" OR "mobile health" OR "remote consultation" OR "videoconferencing" OR "teleconsultation" OR "tele monitoring" OR "tele rehabilitation") AND ("quality of life" OR "life quality" OR "health status" OR "health level” OR "level of health" OR "sickness impact profile" OR "acuity" OR "severity of illness" OR "illness severity" OR "patient outcome assessment” OR "patientcentered outcome" OR "process assessment" OR “clinical copd questionnaire" OR “chq” OR “sgrq").

Publications were included in this review if they fulfilled the following inclusion criteria: 1) original research,
2) published in year 2000 or later, 3) specific reporting on QoL, and 4) consistent and rigorous methodology, including transparent management of data and explicit considerations on how to quantify outcomes on QoL. Publications were not included based on the following exclusion criteria: 1) nonEnglish papers, 2) extremely small populations $(n<30)$ or case studies, and 3) mixed population with non-COPD patients (further details are given in Figure 1). A metaanalysis of included studies was not performed, primarily due to the limited number of published studies fulfilling the inclusion criteria.

\section{Results}

Based on title and abstract content, 47 articles were identified for further evaluation. These articles were either excluded from or included in the present analysis based on the inclusion and exclusion criteria described previously (Figure 1); most of the excluded articles fulfilled several exclusion criteria. Ultimately, a total of 18 original articles

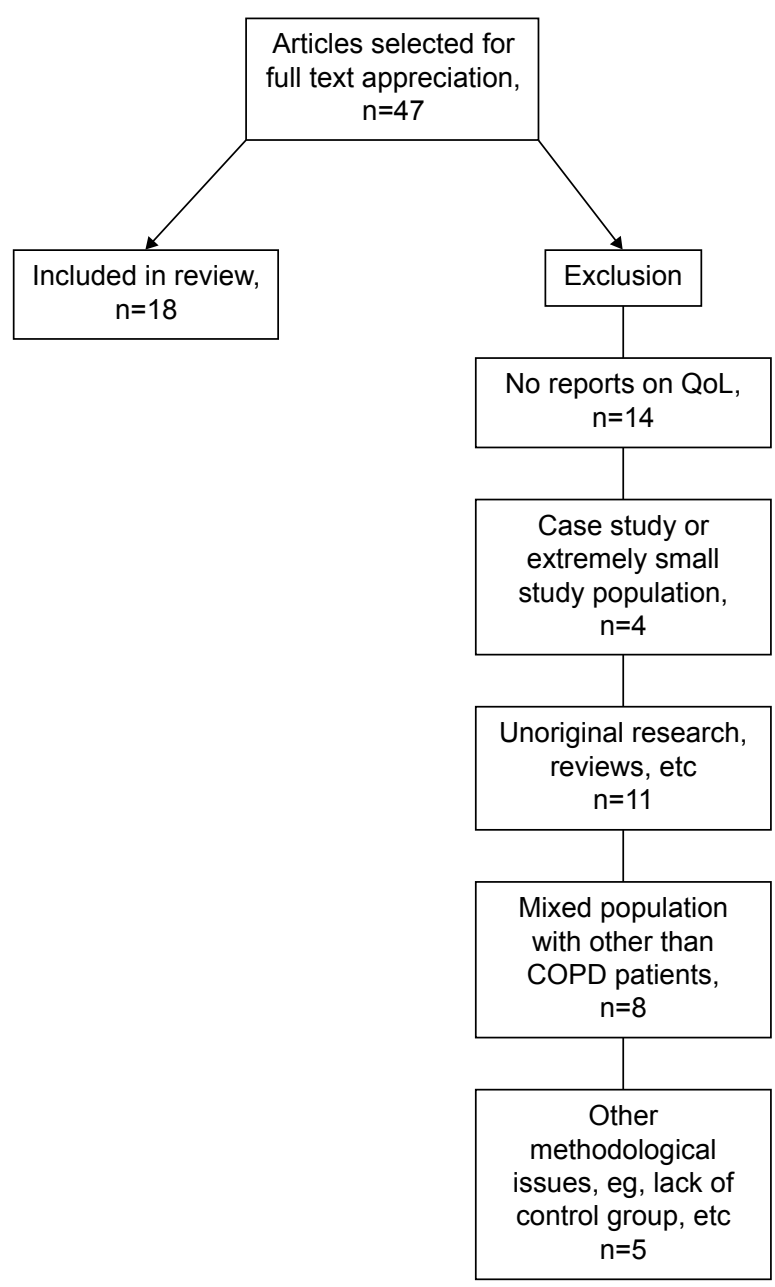

Figure I Selection process. Abbreviation: QoL, quality of life. 
were included in this review, as they fulfilled all criteria. In order to facilitate the overview, the included articles were arbitrarily divided according to the number of patients completing each study as "larger studies" (data on $>100$ patients at follow-up) and "smaller studies" (data on $<100$ patients at follow-up). An overview of the studies included in this review is given in Table 1 (larger studies) and 2 (smaller studies), respectively.

\section{Larger studies}

A total of six studies (comprising 1,017 patients) addressing the effect of various telemedical interventions were identified and included in this review.

In a two-center, randomized controlled clinical trial, published in 2015, McDowell et $\mathrm{al}^{9}$ provided all patients, 55 in the intervention group and 55 patients in the control group, with a standardized home-based program of specialist respiratory assessment, which entailed COPD education, including recognition of symptoms of exacerbations, advice on smoking cessation, and review of self-management techniques (Table 1). In addition to the home-based program, patients in the intervention group received a home telehealth device that recorded data on vital signs and asked questions related to symptoms to which a "yes" or "no" was the possible answer. A project nurse reviewed the data and responded to alerts indicating deterioration in health status. Outcomes on QoL were measured with the St George's Respiratory Questionnaire (SGRQ), the EuroQol-5 Dimension Questionnaire (EuroQol-5D), and the Hospital Anxiety and Depression Scale (HADS). At 6 months, total SGRQ scores had improved in the intervention group as compared to the control group, with a statistically significant difference between the groups that was greater than the minimal clinically important difference (MCID) (mean 61.1 vs 66.8, $P$-value $=0.001$ ). The same pattern was seen for the SGRQ subscores: the symptoms score improved in the intervention group relative to the control group (mean 71.5 vs $79.0, P$-value $=0.002$ ); the activity score improved in the intervention group relative to the control group (mean 77.8 vs $82.8, P$-value $=0.003$ ); the impact score improved in the intervention group relative to the control group (mean 47.7 vs 53.0, $P$-value $=0.03$ ), with all subscores showing statistically significant differences between the groups. However, in contrast to these findings, the EuroQol-5D scores, including the visual analog scale, improved in the control group but declined in the intervention group, although the betweengroup differences did not reach statistical significance. The HADS anxiety scores revealed an improvement in the intervention group relative to the control group, with a statistically significant difference between the groups (mean 7.15 vs $8.81, P$-value $=0.01$ ). In line with this, the HADS depression scores revealed an improvement in the intervention group relative to the control group but with a statistically nonsignificant difference between the groups ( mean 6.87 vs 7.50, $P$-value $=0.29)$. Several aspects of QoL were evaluated by standardized measures, and most of them revealed a positive impact on QoL in favor of the telehealth care intervention, possibly because the intervention included, compared to other studies, close monitoring.

In 2014, a dual-site randomized clinical trial was published by Blumenthal et $\mathrm{al}^{3}$ who reported comparable ambivalent results based on a different mode of intervention. A total of 298 patients (147 and 151, respectively, in the intervention and control groups), completed the study, including a posttreatment assessment after 16 weeks. Outcomes were measured using the Beck Depression Inventory-II, the State-Trait Anxiety Inventory-State, the Short Form-36 (SF-36), the Pulmonary Quality of Life Scale, the University of California, San Diego (UCSD) Shortness of Breath Questionnaire, the Brief Fatigue Inventory, and the SGRQ. The intervention consisted of extensive instructions in cognitive-behavioral coping skills delivered by phone to patients and partners on a weekly basis in 30-minute sessions. The control group patients and partners also received weekly COPD education by phone, focusing on topics relevant to COPD but without providing instructions on specific coping strategies. As such, both groups interestingly received some degree of telemedical intervention. In their analysis, the authors reported their results by making an overall distinction between so called "psychological" QoL and "somatic" QoL. Overall, the intervention group made greater improvements in psychological QoL than the control group, attaining less depression (Cohen's $d=0.22,95 \%$ confidence interval $[C I]=0.08-0.36, P$-value $=0.002)$, less anxiety $(d=0.17$, $95 \% \mathrm{CI}=0.02-0.33, P$-value $=0.030)$, better overall mental health $(d=0.17,95 \% \mathrm{CI}=0.03-0.32, P$-value $=0.021)$, better emotional role functioning $(d=0.29,95 \% \mathrm{CI}=0.10-0.48$, $P$-value $=0.003)$, and better social functioning $(d=0.21$, $95 \% \mathrm{CI}=0.03-0.38, P$-value $=0.023$ ). Additionally, it was observed that intervention group patients with lower psychological QoL at baseline attained the greatest improvements compared to the control group. There were also improvements, with varying statistical significance, in the intervention group relative to the control group in terms of somatic QoL, with less fatigue $(d=0.34,95 \% \mathrm{CI}=0.18-0.50$, $P$-value $=0.0001)$, less shortness of breath on the SGRQ 


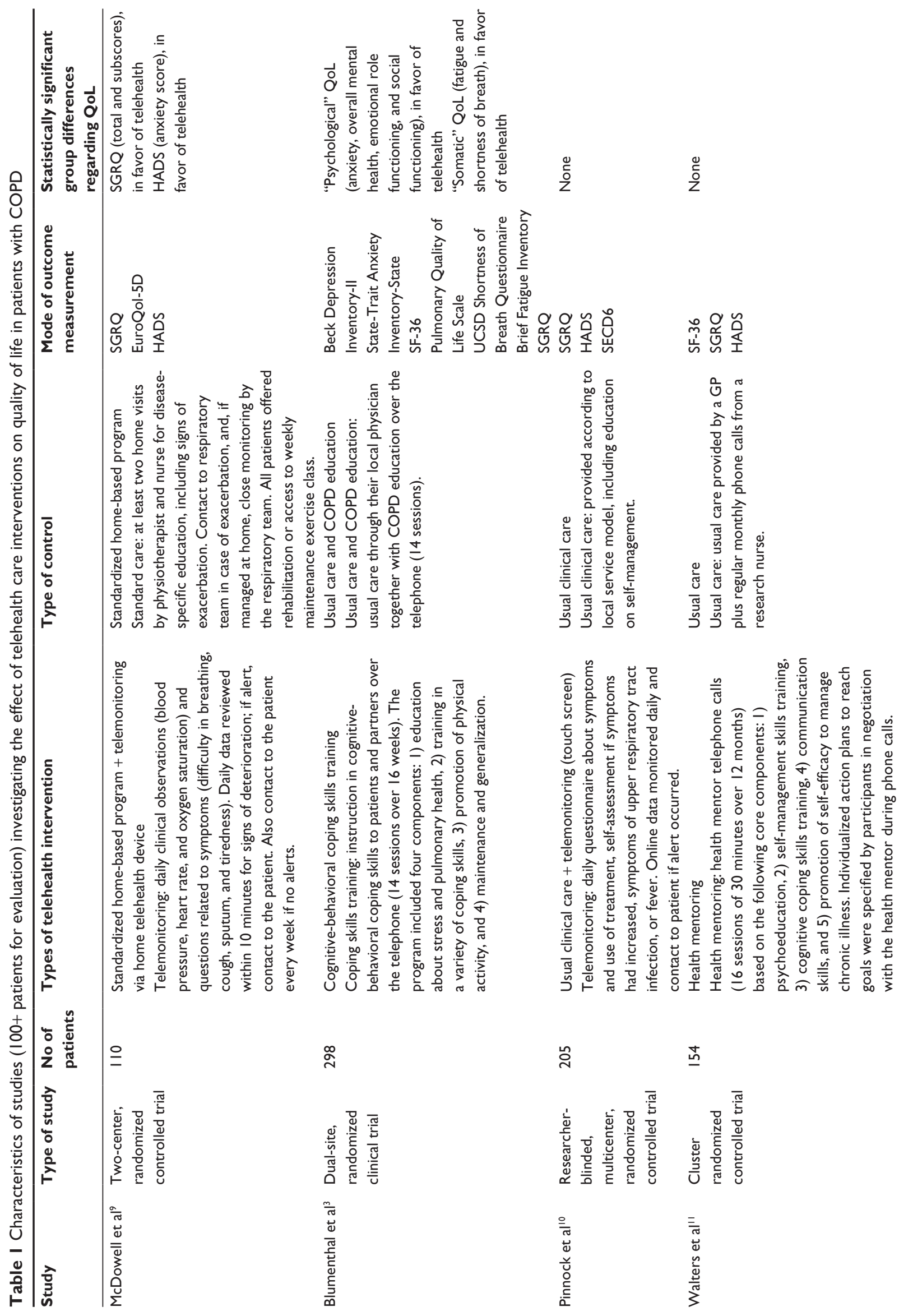




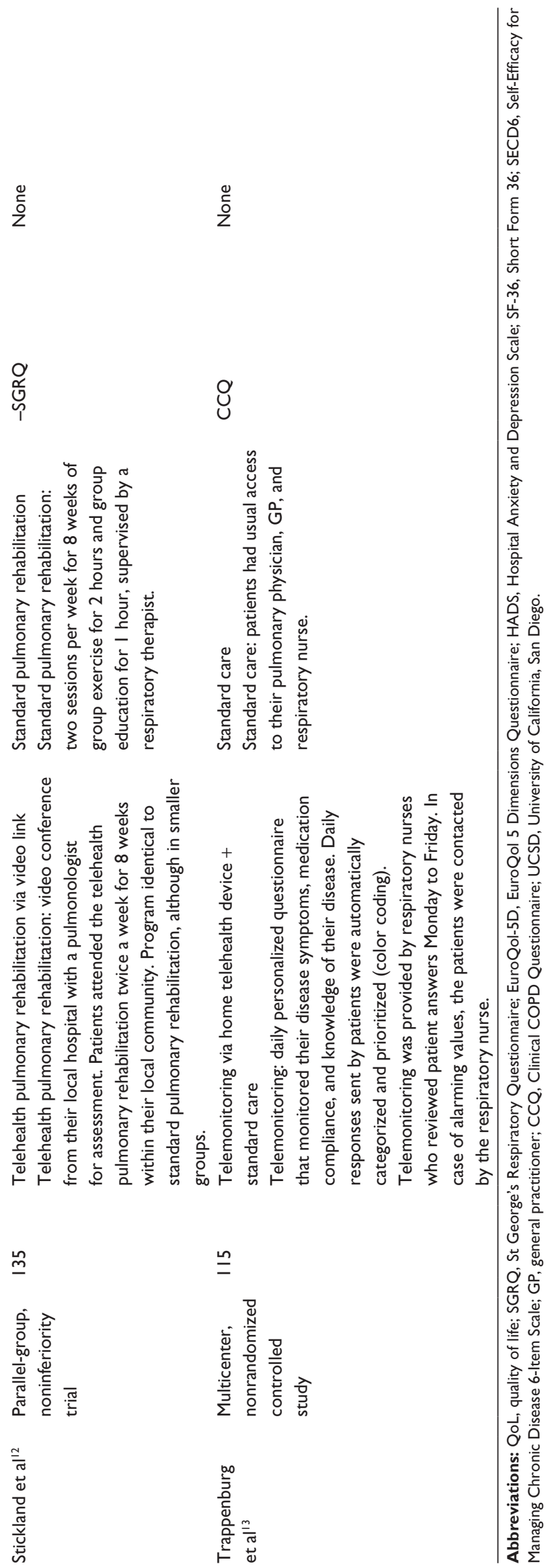

$(d=0.11,95 \% \mathrm{CI}=-0.01-0.23, P$-value $=0.68)$ but not on the UCSD Shortness of Breath Questionnaire $(d=-0.01,95 \%$ $\mathrm{CI}=-0.12-0.10, P$-value $=0.859)$, and improvement in the Pulmonary Quality of Life Scale $(d=0.13,95 \%$ CI $=0.01-0.24$, $P$-value $=0.040$ ). The MCID of four points was only clearly reported to have been exceeded in the intervention group when looking at the SF-36 emotional role functioning score and the adjusted posttreatment score for the SGRQ. No group differences were seen for the SF-36 pain, physical role, general health, or physical functioning scores.

A 12-month, researcher-blinded, multicenter, randomized controlled trial by Pinnock et al, ${ }^{10}$ comprising 105 patients in a telemonitoring plus usual care group and 100 patients in the control group completing the final questionnaires, was published in 2013 (Table 1). The control group received usual clinical care, including self-management advice, the only difference between the groups thus being the telemonitoring. Information on changes in health-related QoL was collected using the SGRQ, the HADS, and the Self-Efficacy for Managing Chronic Disease 6-Item Scale (SECD6). The telemonitoring consisted of a simple touch screen device which patients used to record symptoms and treatment use and to monitor oxygen saturation. The information was sent to the supporting clinical team, who were alerted if readings were omitted or breached thresholds. The study revealed that $25 \%$ of participants in both groups experienced an improvement by more than four units (MCID) in their SGRQ score. However, no significant differences between groups were found in any of the questionnaire scores, and neither were any significant differences from baseline to 1 year found in either group.

In a cluster randomized controlled trial by Walters et $\mathrm{al}^{11}$ published in 2013, 74 patients completed a telemedically based health mentoring program. The intervention consisted of 16 scheduled 30-minute phone calls over 12 months, focusing on psychoeducational training, self-management skills, cognitive coping skills, communication skills, and self-efficacy in illness management. A total of 80 patients in the control group received usual care plus monthly phone calls without any psychological advice or skills training. Outcomes related to QoL were measured using the SF-36 Health Survey, SGRQ, and HADS at baseline, 6 months, and 12 months. The telemedical intervention did not produce any statistically significant improvements on either of these scales when compared to the control group. However, both groups experienced a statistically significant decrease in anxiety as measured by the HADS.

With a different perspective and alternative implementation, Stickland et al, ${ }^{12}$ in a 2011 parallel-group, noninferiority 
trial with a 6-month follow-up, looked at delivering pulmonary rehabilitation by means of telemedicine. A total of 88 patients from the standard rehabilitation program and 47 patients from the telehealth rehabilitation program provided data at 6-month follow-up, the outcomes on QoL being measured with the SGRQ. The control group received standard pulmonary rehabilitation at a main training center twice a week for 8 weeks, with supervised sessions including exercise and selfmanagement education. The same pulmonary rehabilitation program was delivered to patients in the telehealth group gathering in smaller groups at satellite locations, the same sessions being delivered via video link and supervised by local staff. Overall, clinically and statistically significant improvements were found in QoL as measured by SGRQ subscores and total scores in both groups. For the telehealth group, total SGRQ score before pulmonary rehabilitation was $50.9(\mathrm{SD}= \pm 16.2)$, and at 6-month follow-up, it was 44.6 ( $\mathrm{SD}= \pm 15.4$, $P$-value $<0.05$ vs before rehabilitation). For the control group, total SGRQ score before pulmonary rehabilitation was 45.4 ( $\mathrm{SD}= \pm 17.1$ ), and at 6-month follow-up, it was 38.1 ( $\mathrm{SD}= \pm 18.2, P$-value $<0.05$ vs before rehabilitation). However, no statistically significant differences were found between the two groups. This study seems to demonstrate that rehabilitation may be delivered as a telemedical intervention without a loss of benefit with regard to QoL. However, further studies are needed to investigate whether this setup has the potential to improve QoL, and preferably in studies with a much higher completion rate.

Finally, a 6-month multicenter nonrandomized controlled study from 2008 by Trappenburg et $\mathrm{al}^{13}$ compared telemonitoring to standard care. A total of 59 patients in the intervention group and 56 patients in the control group provided data on QoL as measured by the Clinical COPD Questionnaire (CCQ) at baseline and after 6 months. Patients in the intervention group were monitored by using a small device that requested daily answers to questions on symptoms, medication compliance, and disease knowledge, as well as providing direct feedback to the user. Uploaded data were monitored by respiratory nurses who, if needed, could contact a pulmonary physician or clinic. At 6-month follow-up, no significant changes in QoL related to CCQ scores were observed within groups, nor between groups. The relatively limited monitoring, and only on weekdays, may have had important impact on the findings.

\section{Smaller studies}

A total of 12 studies (comprising 619 patients) addressing the effect of a variety of telemedical interventions were identified and included in this review (Table 2).
In a recent two-center, noninferiority, randomized controlled trial by Jakobsen et al, ${ }^{14}$ published in 2015 , the patients were included around the time of a hospital admission for exacerbation of COPD. The 29 patients in the intervention group were discharged from hospital within 24 hours of admission and equipped with a touch screen with webcam and functions for measuring vital signs, as well as with a nebulizer, an oxygen compressor, and a box with relevant medication (Table 2). Daily ward rounds were performed using the touch screen at appointed hours, and hospital personnel were instructed to treat the intervention group patients like patients present at the hospital, except for the physical contact. Patients in the control group were hospitalized and received standard hospital care for an exacerbation. Outcomes on QoL were measured using the SGRQ, the CCQ, and the EuroQol-5D. Data were collected at baseline, during the intervention, and at follow-up visits 30 days, 90 days, and 180 days after discharge. Considering the entire period, improvements in both SGRQ, CCQ, and EuroQol-5D scores were found in both groups only within the first 30 days after discharge, but these improvements were statistically insignificant. Similarly, no statistically significant differences in scores were found between the groups. This pilot study showed that early discharge combined with telehealth care may be feasible for patients with acute exacerbation of COPD without having negative impact on the patients' QoL. Further studies are needed to address whether this intervention has the potential to improve QoL for COPD patients in relation to exacerbations.

In 2014, Berkhof et $\mathrm{al}^{15}$ published observations from their single-center prospective randomized controlled trial comprising 95 patients. The enrolled patients were randomized to either telehealth care $(n=50)$ or usual care for 6 months. The outcome of the intervention was assessed by the CCQ, the SGRQ, and the SF-36. The intervention consisted of nurses performing phone calls that followed a predetermined structure with a short introductory conversation followed by administration of the CCQ, every 2 weeks (Table 2). In case CCQ scores exceeded the MCID compared to the previous score, a pulmonologist would contact the patient to inquire about symptoms of an exacerbation. The study found that this type of intervention resulted in a statistically nonsignificant unfavorable increase in CCQ total score of $0.14 \pm 0.14$ after 6 months for the intervention group, contrasting with a favorable decrease of $-0.03 \pm 0.14$ for the control group (difference $0.17 \pm 0.19,95 \% \mathrm{CI}=-0.21-0.55, P$-value $=0.38)$. Similarly, an increase in SGRQ total score of $6.7 \pm 1.8$ for the intervention group and $4.3 \pm 1.9$ for the control group (difference $2.4 \pm 2.6,95 \% \mathrm{CI}=-2.7-7.5, P$-value $=0.36)$, with activity 

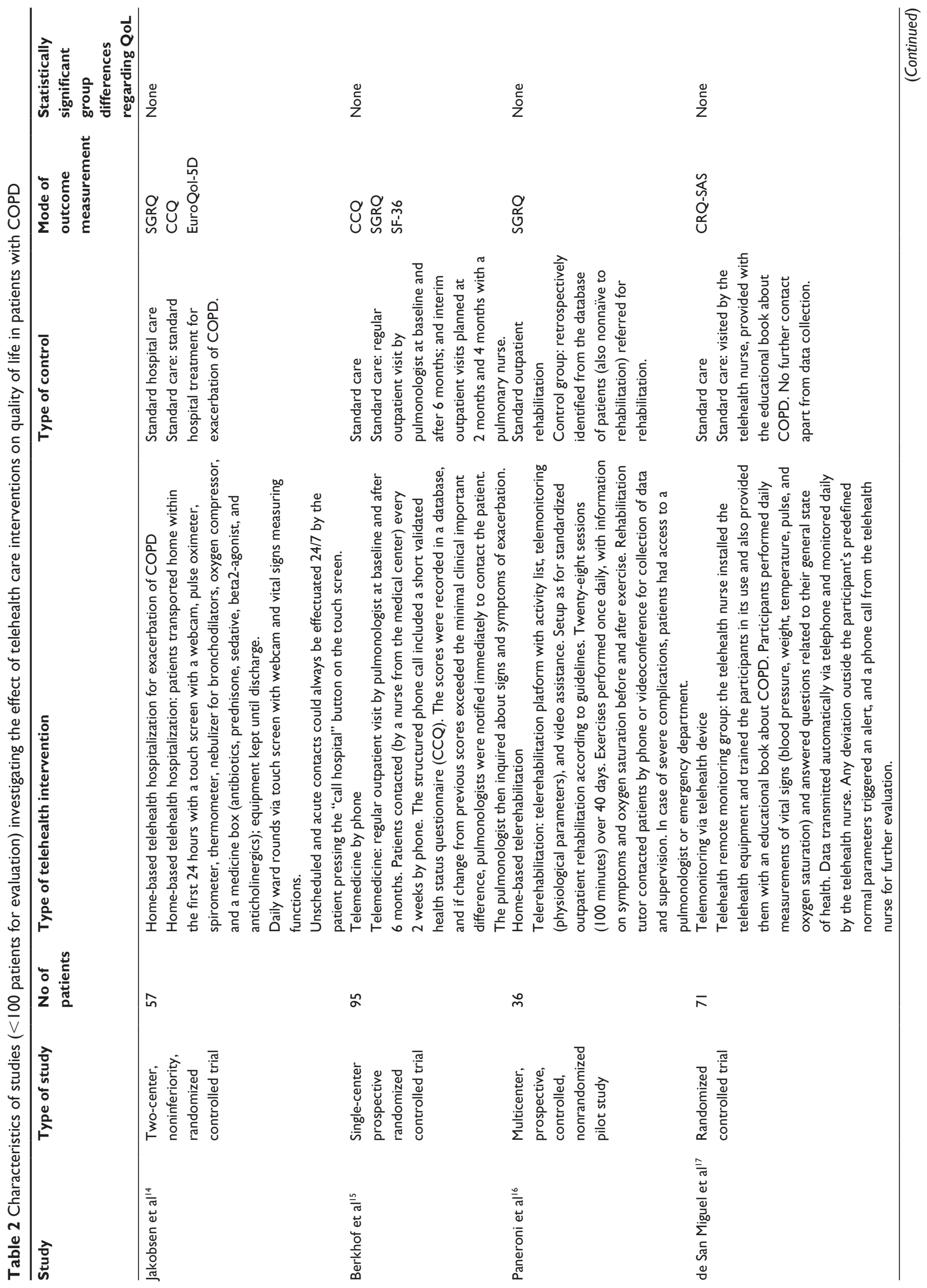


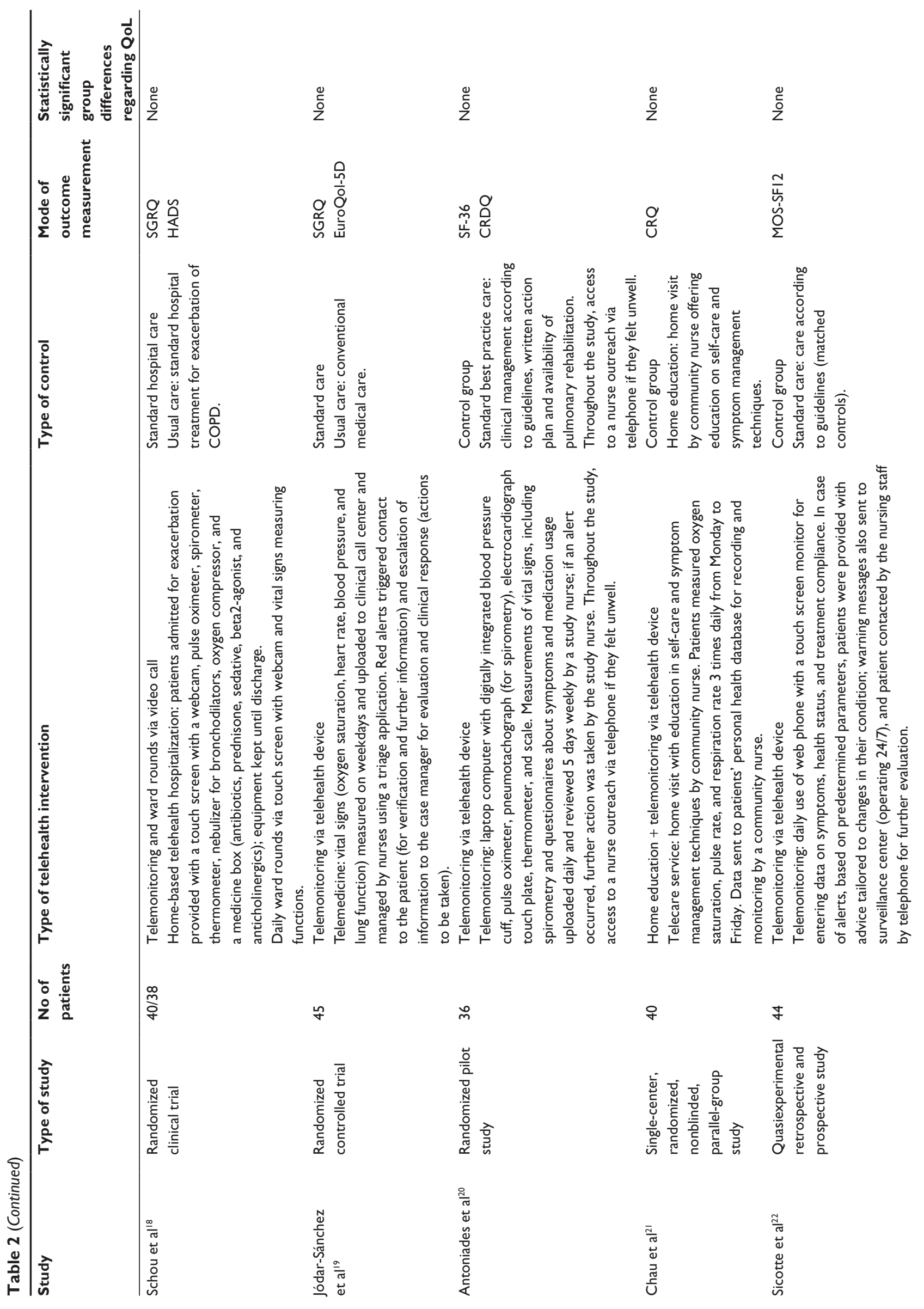




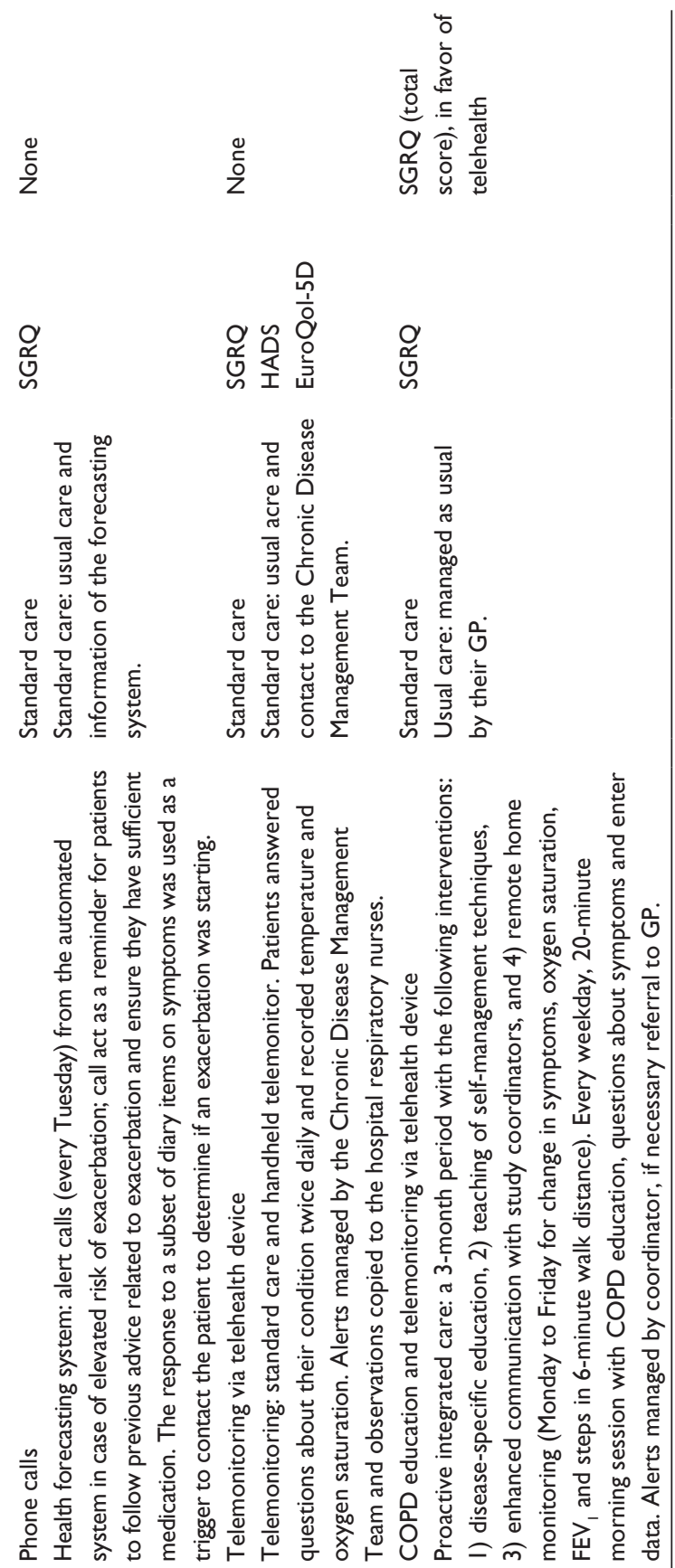

$\hat{N}$

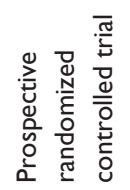

웅

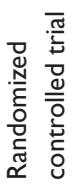

$\stackrel{\infty}{m}$

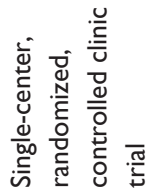

$\frac{\sqrt{\frac{\pi}{0}}}{\frac{0}{0}}$ and impact subscores relatively favoring the control group. The SF-36 score results were inconsistent. The observations of no positive impact on QoL in this study may be due to the very limited telehealth care intervention.

Yet, in another type of setup, a multicenter, prospective, controlled, nonrandomized pilot study published in 2014 by Paneroni et $a 1^{16}$ compared the implementation of a rehabilitation program by way of a home-based telemedical program versus a standard outpatient program, each group including 18 patients. The rehabilitation program consisted of 28 sessions performed during a maximum period of 40 days (Table 2). Outcomes on QoL were measured using the SGRQ at baseline and at the end of the rehabilitation program. Both groups completed the same rehabilitation program with monitoring of physiological values, performing physical exercises, being supervised during exercises, and receiving COPD education, the primary difference being that the telemedicine group transmitted physiological data wirelessly and received supervision as well as education via a videoconference in their own home instead of in a face-to-face outpatient setting (Table 2). The two types of rehabilitation setup produced an improvement in SGRQ scores in both groups by more than the MCID with $-6.9(\mathrm{SD}= \pm 9.96, P$-value $=0.009)$ for the teleintervention group and -9.9 ( $\mathrm{SD}= \pm 12.92$, $P$-value $=0.007)$ for the control group. Importantly, however, no statistically significant differences in outcome were observed between the two groups, and the improvement observed was actually greater in the control group than in the intervention group.

In a narrower approach, using only the Chronic Respiratory Disease Questionnaire Self-Administered Standardized (CRQ-SAS) to assess changes in QoL, de San Miguel et al ${ }^{17}$ reported similar results in 2013, based on a study of 36 patients in the telehealth group and 35 in the control group completing a 6-month randomized controlled trial. Both groups received a book with information about COPD, while the intervention group additionally was taught to measure and record vital signs daily using a small portable unit. Data were transmitted to a nurse who would contact the patient in case of an alert. A clinically significant positive change (2 point score change) was found within the intervention group for the mastery domain, expressing patients' feeling of control over their disease. However, no statistically significant differences were found between the groups for any of the domains at baseline or at 6 months. Although using only limited tools to assess impact on QoL, some positive observations were found, which call for further studies.

Another randomized clinical trial by Schou et al, ${ }^{18}$ published in 2013, looked at the effect of telemedicine 
on QoL in a more acute setting. Patients admitted to an emergency department for an exacerbation of COPD were randomized to continuing treatment at home using telemedicine or continuing treatment in hospital. Outcomes related to QoL were measured using the SGRQ, with 21 patients in the intervention group and 19 patients in the control group providing data at follow-up after 3 months, and the HADS, with 20 patients in the intervention group and 18 patients in the control group providing data at follow-up after 3 months. The telemedical intervention lasted only 2-8 days and consisted in patients receiving a touch screen PC, devices for monitoring vital signs, as well as oxygen, a nebulizer, and additional medicine. The touch screen enabled the patients to connect to a call center to ask for help or advice in case of acute dyspnea, in addition to daily video calls being made by the call center staff. At 3-month follow-up, both groups had improved all their SGRQ scores beyond the cutoff for MCID as compared to 3 days after discharge, but there was no statistically significant difference in scores between the groups. Thus, 3 days after discharge, the total SGRQ scores for the intervention group and control group were $64(\mathrm{SD}= \pm 17)$ and $58(\mathrm{SD}= \pm 14)$, respectively, with a $P$-value $=0.29$, and at 3 -month follow-up, the corresponding scores were $55(\mathrm{SD}= \pm 19)$ and $48(\mathrm{SD}= \pm 19)$, respectively, with a $P$-value $=0.19$. The HADS score also did not show statistically significant differences between the groups, and neither group improved their HADS score at follow-up.

In 2013, Jódar-Sánchez et al ${ }^{19}$ published a study including 45 patients in a 4-month randomized controlled trial with 24 patients in the intervention group and 21 patients in the control group being analyzed. Outcomes on QoL were measured with the SGRQ and the EuroQol-5D at the beginning and end of the trial period. The intervention consisted of patients on weekdays registering and transmitting vital signs to a call center, where the staff would react to readings falling outside of predetermined parameters. The control group received standard care. The study found clinically important improvements in SGRQ score for both groups, in all domains for the intervention group and only in total score for the control group. The total SGRQ scores at follow-up had changed $-10.9(\mathrm{SD}= \pm 21.9)$ for the intervention group and $-4.5(\mathrm{SD}= \pm 19.7)$ for the control group, with a $P$-value $=0.53$. No statistically significant improvements were found for either SGRQ or EuroQol-5D scores for any of the two groups.

Antoniades et $\mathrm{al}^{20}$ published a 12 -month randomized pilot study in 2012, with 16 patients in the intervention group and 20 patients in the control group completing the full study.
Changes in QoL were measured using the SF-36 and the Chronic Respiratory Disease Questionnaire (CRDQ). While the control group received standard care, patients in the intervention group also recorded their vital signs, symptoms, and medication usage on a daily basis using an easy-to-use laptop computer. Data were uploaded to the research staff, and they were, therefore, able to react to deteriorating parameters. Regarding QoL, no statistically significant differences between baseline and 12-month measurements were found within each group or between the groups for the SF-36 or the CRDQ. However, this pilot study showed the intervention to be feasible.

Yet, in another small feasibility study published in 2012, Chau et $\mathrm{al}^{21}$ had 22 patients in the intervention group and 18 patients in the control group completing a single-center, randomized, nonblinded, parallel-group study over 2 months. Outcomes on QoL were measured with the CRQ. Both the control group and the intervention group received home visits by a community nurse, who offered education on self-care and symptom management. In addition to this, the telemedicine group received a simple kit to monitor vital signs, including oxygen saturation, three times daily on weekdays. Data were afterward uploaded to a platform so a nurse could monitor and react to changes in physiological parameters. No statistically significant changes in CRQ scores within groups or between groups were found between baseline and follow-up. This study confirmed the feasibility of the setup in a slam (limited, uncontrolled) setting, but larger studies are clearly needed.

Another alternative approach, using a quasiexperimental retrospective and prospective design, was used in a study by Sicotte et $\mathrm{al}^{22}$ published in 2011. A total of 22 patients were included in the intervention group with 22 individually matched controls included in the control group. The average intervention period was 4.8 months. Outcomes on QoL were measured using the Medical Outcomes Study 12-Item Short Form Health Survey (MOS-SF12). The telemedical intervention consisted of uploading vital signs to a nursing team using a touch screen telephone. It was used as a kind of patient education and was terminated when it was estimated that the patient had acquired necessary knowledge and adopted desired behaviors. Patients in the control group received standard home care. It was observed that scores related to QoL remained stable for the intervention group, while deteriorating in the control group. This telehealth care educational setup might be useful, but larger randomized trials are needed.

In a prospective randomized controlled trial published in 2011, Halpin et $\mathrm{al}^{23}$ implemented an algorithm aiming 
to predict exacerbations of COPD. For $3 \frac{1}{2}$ months, the 39 patients completing the trial in the intervention group daily answered questions related to their health state and presence of symptoms on a mobile phone. Early indications of an exacerbation would prompt an immediate contact to the patient in question. The 38 patients allocated to the control group received standard care. Outcomes on QoL were measured using the SGRQ. No statistically significant differences between the two groups were found in the SGRQ scores at the end of the study. However, this intervention seems to have potential for being useful in early detection, and perhaps prevention, of exacerbations.

In a randomized controlled trial published in 2010, Lewis et $\mathrm{al}^{24}$ had 20 patients in an intervention group and 20 patients in a control group, providing data on QoL from the SGRQ, the HADS, and the EuroQol-5D. All patients completed a pulmonary rehabilitation program, and data on QoL were extracted before the program, after the program, and periodically during the trial. During the trial, patients in the control group received 12 months of standard care, while patients in the intervention group additionally received a small telemonitor to record vital signs and symptoms twice daily for 6 months followed by 6 months standard care only. Data were monitored by a nursing staff receiving alerts in case of deterioration. The statistical analysis found that the SGRQ and HADS scores improved immediately following pulmonary rehabilitation, but no differences in QoL related to these scores were found during the trial. EuroQol-5D scores were only collected during the trial, at 0 week, 4 weeks, 25 weeks, 30 weeks, and 52 weeks, but these also failed to show any between-group differences in QoL.

In a small single-center, randomized, controlled clinical trial published in 2009, with 19 patients in the intervention group and 19 patients in the control group completing a 3-month intervention period, Koff et $\mathrm{al}^{25}$ assessed the effects of a diversified telemedical intervention, which both provided COPD-specific education on an electronic interface and retrieved answers to questions on symptoms and data on vital signs (Table 2). Furthermore, the system could also test patient knowledge, assess medical adherence, and probe for the presence of depression. Patients in the control group received standard care. In case the system registered a worsening of condition, the coordinator contacted the patient, and, if necessary, would contact the patient's primary care physician. The SGRQ was used to measure outcomes in relation to QoL. At 3 months, individual SGRQ scores improved statistically nonsignificantly in favor of the intervention group. The symptom score decreased by 12.8 units in the intervention group compared with 3.3 units in the control group $(P=0.27)$; the activity score decreased by 8.8 units in the intervention group compared with 0.5 units in the control group $(P=0.16)$; and the impact score decreased by 6.6 units in the intervention group compared with 0.6 units in the control group $(P=0.20)$. However, the total SGRQ score improved statistically significantly in favor of the intervention group by decreasing 10.3 units compared to 0.6 units in the control group $(P=0.018)$. The positive impact of this telecare intervention on QoL may be due to the combination of education, including self-management skills, and close monitoring of several objective parameters.

\section{Discussion}

\section{Summary}

Three out of 18 studies (comprising a total of 1,636 patients) fulfilling the criteria for being included in this review found statistically significant improvements in QoL in favor of the telemedical intervention group; one study found statistically insignificant differences in favor of the intervention group; one study found a statistically insignificant difference in favor of the control group; and finally, 13 studies found no differences in QoL between the groups.

\section{Different modes of telehealth application and different outcomes}

After reviewing this bulk of the literature on the present topic, it becomes evident that telehealth and related terms encompass a variety of instruments and modes of application. The relevance of acknowledging these differences emerges from the concurrent analysis. Generally speaking, the interventions analyzed here can be divided into those that provided a service similar to what was provided to the control group, but by means of some telemedical application; and those that provided a telemedical service in addition to what was provided to the control group. In the first instance, similar services included pulmonary rehabilitation and hospital ward rounds delivered via video link as opposed to outpatient training and in-hospital treatment. In the second instance, additional services could further be divided into those that represented an active intervention, such as the delivery of coping skills training or COPD education by phone or computer, and those that represented a passive intervention, such as vital signs and symptoms monitoring; both active and passive interventions are usually being compared to standard care in the control groups.

In terms of QoL in COPD patients, telehealth is no holy grail, seeing that none of these modes of application, 
as considered in this review, unequivocally produced any distinctly recognizable or statistically significant improvements in QoL. However, some interesting and important observations have been made. Although not that convincing, as it was not the case for all of them, only active interventions were able to produce statistically significant improvements in QoL in the intervention groups compared to the respective control groups. The interventions described in the studies by McDowell et $\mathrm{al}^{9}$ and Blumenthal et $\mathrm{al}^{3}$ were similarly active by delivering some form of coping or self-management skills training without leaving the control groups completely "out in the dark"; that is, the control groups in both studies received some degree of COPD education. As it has been suggested elsewhere, ${ }^{26}$ not having "pure" control groups receiving only standard care may have contributed to weakening the statistical power of the study outcomes, but both studies nonetheless observed statistically significant improvements in QoL in the intervention groups as compared to the control groups. In a more straightforward fashion, Koff et al ${ }^{25}$ observed similar results, though without specific coping skills training, in a study with a control group receiving only standard care. These studies may suggest that active interventions are the way to go in terms of improving QoL in telemedical interventions. Yet, doubt is cast on this hypothesis by the study conducted by Walters et $\mathrm{a}^{11}$ in which the full arsenal of coping and self-management skills training was put to use in the intervention group and the control group that received only phone calls without any form of training in addition to the standard care. Here, no statistically significant differences were found between the groups in terms of QoL.

\section{Success criteria for telehealth revised}

Perhaps a different kind of question has to be asked: Is it at all reasonable to expect, or even demand, an improvement in QoL from the implementation of telehealth? Is it a realistic success criterion? Among the studies considered here, those that examined the use of telemonitoring in addition to standard care all failed at improving QoL in the intervention group compared to the control group, as did telemedical pulmonary rehabilitation as compared to standard outpatient pulmonary rehabilitation and telemedical treatment for exacerbations of COPD as compared to standard in-hospital treatment. Nonetheless, it is important to note that none of the studies observed a significant decrease in QoL in the intervention groups when compared to the control groups. A few details deserve to be highlighted to add further nuance. First, in the study performed by Blumenthal et al, ${ }^{3}$ it was observed that the greatest potential for improvements in $\mathrm{QoL}$ was in the group of patients with the lowest QoL score at baseline. Hence, it may be speculated that patients with severe COPD will benefit more from active telemedical interventions than other groups of COPD patients, but it is a hypothesis that needs to be further investigated in future studies by classifying patients according to disease severity. Second, Sicotte et $\mathrm{al}^{22}$ observed that scores on QoL deteriorated in the control group while remaining stable in the intervention group. This supports the argument that unchanged QoL scores be accepted as a relative success. Third, the study by Berkhof et a ${ }^{15}$ found statistically insignificant decreases in QoL scores in an intervention group offered no other form of intervention than the CCQ questionnaire. This finding suggests that not just any kind of extra attention in the form of a telemedical intervention is beneficial or neutral. Insofar as telehealth performs better in other outcomes than QoL, attaining results comparable to those of standard care may, as already proposed, be regarded as a relative success. As such, the case for using telehealth for patients with COPD is not strong if only backed by data on QoL, but it also does not provide an argument against the use of telehealth. On the contrary, when looking at QoL as an outcome, the data indicate that usual care may well be substituted by comparable telehealth solutions. As mentioned previously, for this argument to be fully valid, some other advantages must follow from the use of telehealth. The extended literature on telehealth and COPD does not provide any unambiguous answers. Risks of hospitalizations, ${ }^{1,6,27}$ risks of exacerbations, ${ }^{6,27}$ risks of visiting the emergency department, ${ }^{6,27}$ health care costs, ${ }^{1}$ and duration of bed days ${ }^{6}$ have only sporadically been found to be lower in telemedical intervention groups as compared to control groups. Therefore, clear evidential support for the promise of telehealth as a more cost-effective treatment that can simultaneously add to the number of quality-adjusted life years in patients is still lacking. Be that as it may, the total amount of data on telehealth and COPD is still limited because of the short time in which it has been relevantly collected, and the current conclusions can only be considered to be preliminary. Consequently, future research is necessary to clarify not only the outcomes on QoL but also all outcomes following the implementation of telehealth in patients with COPD.

\section{Conclusion and future directions}

Different modes of telehealth application have been indicated to influence outcomes on QoL in COPD patients in dissimilar ways. It is reasonable to think that the case could be the same for other outcomes and for other types of patients, but this needs to be tested in future studies on telehealth. In doing so, 
greater care is needed in emphasizing the differences between the different modes of application to directly examine any potential causality.

Another indication that warrants further research is the possibility of dissimilar outcomes in different groups of COPD patients, stratified by severity, with one study ${ }^{3}$ suggesting that patients with a lower QoL at baseline experience greater improvements in this outcome when compared to patients with a better baseline QoL score. Future studies must consider if this is a relevant trend, regarding both the outcome on QoL and the other outcomes.

Finally, after considering the results presented here, a discussion of the relevance of improvement in QoL as a success criterion should follow, entailing a comparison to absence of deterioration, relative to control groups, as a perhaps more realistically acceptable success criterion.

\section{Strengths and limitations}

- Our systematic review shows only limited evidence for a positive effect of telemedical interventions on QoL in patients with COPD.

- A quantitative synthesis was not possible due to the heterogeneity of the relatively low number of available studies.

\section{Author contributions}

CSU conceived and designed the study. TLG drafted the paper. All authors contributed toward data analysis, drafting and critically revising the paper and agree to be accountable for all aspects of the work.

\section{Disclosure}

The authors report no conflicts of interest in this work.

\section{References}

1. Cruz J, Brooks D, Marques A. Home tele monitoring effectiveness in COPD: a systematic review. Int J Clin Pract. 2014;68(3): 369-378.

2. Bentley CL, Mountain GA, Thompson J, et al. A pilot randomized controlled trial of a Telehealth intervention in patients with chronic obstructive pulmonary disease: challenges of clinician-led data collection. Trials. 2014;15:313.

3. Blumenthal JA, Emery CF, Smith PJ, et al. The effects of a telehealth coping skills intervention on outcomes in chronic obstructive pulmonary disease: primary results from the INSPIRE-II study. Psychosom Med. 2014;76(8):581-592.

4. Gudmundsson G, Gislason T, Jansson C, et al. Risk factors for rehospitalisation in COPD: role of health status, anxiety and depression. Eur Respir J. 2005;26(3):414-419.

5. Voncken-Brewster V, Tange H, Moser A, Nagykaldi Z, de Vries H, van der Weijden $T$. Integrating a tailored e-health self-management application for chronic obstructive pulmonary disease patients into primary care: a pilot study. BMC Fam Pract. 2014;15:4.
6. Kamei T, Yamamoto Y, Kajii F, Nakayama Y, Kawakami C. Systematic review and meta-analysis of studies involving telehome monitoringbased telenursing for patients with chronic obstructive pulmonary disease. Jpn J Nurs Sci. 2013;10(2):180-192.

7. Nici L, Zuwallack RL. Pulmonary rehabilitation: future directions. Clin Chest Med. 2014;35(2):439-444.

8. Moher D, Liberati A, Tetzlaff J, et al. Preferred reporting items for systematic reviews and meta-analyses: the PRISMA statement. Open Med. 2009;3(3):123-130.

9. McDowell JE, McClean S, FitzGibbon F, Tate S. A randomised clinical trial of the effectiveness of home-based health care with telemonitoring in patients with COPD. J Telemed Telecare. 2015;21(2):80-87.

10. Pinnock H, Hanley J, McCloughan L, et al. Effectiveness of telemonitoring integrated into existing clinical services on hospital admission for exacerbation of chronic obstructive pulmonary disease: researcher blind, multicentre, randomised controlled trial. BMJ. 2013;347: f6070.

11. Walters J, Cameron-Tucker H, Wills K, et al. Effects of telephone health mentoring in community-recruited chronic obstructive pulmonary disease on self-management capacity, quality of life and psychological morbidity: a randomized controlled trial. BMJ Open. 2013;3(9): e003097.

12. Stickland M, Jourdain T, Wong EY, Rodgers WM, Jendzjowsky NG, Macdonald GF. Using Telehealth technology to deliver pulmonary rehabilitation in chronic obstructive pulmonary disease patients. Can Respir J. 2011;18(4):216-220.

13. Trappenburg JC, Niesink A, De Weert-van Oene GH, et al. Effects of telemonitoring in patients with chronic obstructive pulmonary disease. Telemed J E Health. 2008;14(2):138-146.

14. Jakobsen AS, Laursen LC, Rydahl-Hansen S, et al. Home-based telehealth hospitalization for exacerbation of chronic obstructive pulmonary disease: findings from "the virtual hospital" trial. Telemed JE Health. 2015;21(5):364-373.

15. Berkhof FF, van den Berg JW, Uil SM, Kerstjens HA. Telemedicine, the effect of nurse-initiated telephone follow up, on health status and healthcare utilization in COPD patients: a randomized trial. Respirology. 2015; 20(2):279-285.

16. Paneroni M, Colombo F, Papalia A, et al. Is telerehabilitation a safe and viable option for patients with COPD? A feasibility study. COPD. 2015;12(2):217-225.

17. de San Miguel K, Smith J, Lewin G. Telehealth remote monitoring for community-dwelling older adults with chronic obstructive pulmonary disease. Telemed J E Health. 2013;19(9):652-657.

18. Schou L, Ostergaard B, Rydahl-Hansen S, et al. A randomised trial of telemedicine-based treatment versus conventional hospitalisation in patients with severe COPD and exacerbation - effect on self-reported outcome. J Telemed Telecare. 2013;19(3):160-165.

19. Jódar-Sánchez F, Ortega F, Parra C, et al. Implementation of a telehealth programme for patients with severe chronic obstructive pulmonary disease treated with long-term oxygen therapy. $J$ Telemed Telecare. 2013;19(1):11-17.

20. Antoniades NC, Rochford PD, Pretto JJ, et al. Pilot study of remote telemonitoring in COPD. Telemed $J$ E Health. 2012;18(8): 634-640.

21. Chau JP, Lee DT, Yu DS, et al. A feasibility study to investigate the acceptability and potential effectiveness of a telecare service for older people with chronic obstructive pulmonary disease. Int J Med Inform. 2012;81(10):674-682.

22. Sicotte C, Paré G, Morin S, Potvin J, Moreault MP. Effects of home telemonitoring to support improved care for chronic obstructive pulmonary diseases. Telemed J E Health. 2011;17(2):95-103.

23. Halpin DM, Laing-Morton T, Spedding S, et al. A randomised controlled trial of the effect of automated interactive calling combined with a health risk forecast on frequency and severity of exacerbations of COPD assessed clinically and using EXACT PRO. Prim Care Respir J. 2011;20(3):324-331, 2 p following 331. 
24. Lewis KE, Annandale JA, Warm DL, Hurlin C, Lewis MJ, Lewis L. Home telemonitoring and quality of life in stable, optimised chronic obstructive pulmonary disease. J Telemed Telecare. 2010;16(5): 253-259.

25. Koff PB, Jones RH, Cashman JM, Voelkel NF, Vandivier RW. Proactive integrated care improves quality of life in patients with COPD. Eur Respir J. 2009;33(5):1031-1038.
26. Kroenke K. Distance therapy to improve symptoms and quality of life: complementing office-based care with telehealth. Psychosom Med. 2014;76(8):578-580

27. McLean S, Nurmatov U, Liu JL, Pagliari C, Car J, Sheikh A. Telehealthcare for chronic obstructive pulmonary disease: cochrane review and meta-analysis. Br J Gen Pract. 2012;62(604):e739-e749.

\section{Publish your work in this journal}

The International Journal of COPD is an international, peer-reviewed journal of therapeutics and pharmacology focusing on concise rapid reporting of clinical studies and reviews in COPD. Special focus is given to the pathophysiological processes underlying the disease, intervention programs, patient focused education, and self management protocols.

\section{Dovepress}

This journal is indexed on PubMed Central, MedLine and CAS. The manuscript management system is completely online and includes a very quick and fair peer-review system, which is all easy to use. Visit $\mathrm{http}: / / \mathrm{www}$. dovepress.com/testimonials.php to read real quotes from published authors.

\footnotetext{
Submit your manuscript here: http://www.dovepress.com/international-journal-of-chronic-obstructive-pulmonary-disease-journal
} 